\title{
THE IMPORTANCE OF PSYCHODYNAMIC APPROACH DURING COVID-19 PANDEMIC
}

\author{
Darko Marčinko ${ }^{1,2}$, Miro Jakovljevićc ${ }^{1,2}$, Nenad Jakšić ${ }^{1}$, \\ Sarah Bjedov ${ }^{1} \&$ Aleksandra Mindoljević Drakulić ${ }^{3}$ \\ ${ }^{1}$ Department of Psychiatry and Psychological Medicine, University Hospital Center Zagreb, Zagreb, Croatia \\ ${ }^{2}$ School of Medicine, University of Zagreb, Zagreb, Croatia \\ ${ }^{3}$ Faculty of Humanities and Social Sciences, University of Zagreb, Zagreb, Croatia
}

received: 30.3.2020;

revised: 4.4.2020;

accepted: 8.4 .2020

\begin{abstract}
SUMMARY
The coronavirus (COVID-19) outbreak was labeled a global pandemic by the WHO in March of 2020. Understanding how crisis influence an individual's reactions to stressful events (and vice versa) is important in order to create meaningful and effective interventions. Our literature search have revealed lack of the papers related to psychodynamic approach to recent crisis. Psychodynamic places a large emphasis on defense mechanisms and unconscious mind, where upsetting feelings, urges, and thoughts that are too painful for us to directly look at are housed. Even though these painful feelings and thoughts are outside of our awareness, they still influence our behavior in many ways. Optimal application of psychodynamic approach offers the frame for acceptance of psychological stress in a more positive way and benefits psychological growth. We believe that including psychodynamic approach in the national public and mental health emergency system will empower Croatia and the world during (and after) COVID-19 pandemic crisis.
\end{abstract}

Key words: psychodynamic - mental health - COVID-19 pandemic - resilience - archetypes - post-traumatic growths

\section{INTRODUCTION}

The coronavirus (COVID-19) outbreak was labeled a global pandemic by the WHO in March 13, 2020 not only affecting physical health, but also individual, family and public mental health (Jakovljevic et al. 2020). A novel coronavirus (COVID-19) has been identified as originating in Wuhan, China. It has widely and rapidly spread in China and several other countries, causing an outbreak of acute infectious pneumonia. The outbreak of COVID-19 in the world has caused public mental health stress, anxiety, panic and behavioral disorders. A pandemic describes an infectious disease where we see significant and ongoing personto-person spread in multiple countries around the world at the same time. The last time a pandemic occurred was in 2009 with swine flu, which experts think killed hundreds of thousands of people. Ebola outbreak (2014) also mobilized mental health system (Shultz 2014). Pandemics are more likely if a virus is brand new, able to infect people easily and can spread from person-to-person in an efficient and sustained way. Coronavirus appears to tick all of those boxes. With no vaccine or treatment that can prevent it yet, containing its spread is vital. In addition to the COVID-19 Croatia, particularly its capital Zagreb was hit in March with the earthquake series, the strongest one 5.7 Richter degrees with catastrophic consequences. From a mental health point, the current pandemic is a great challenge for psychiatry and mental health services. Mental health and psychosocial consequences of this pandemic that is proclaimed as war like crisis situation will be an extreme distress for several groups of people: 1. those who have been directly or indirectly in contact with virus; 2 . those who are fragile with hypersensitivity and high vulnerability to stress and prone to panic reaction; 3 . those who were stigmatized, discriminated and chicaned due positive tests and their families; 4. those who lost beloved family members and dear friends due COVID-19; 5. those who lost their home due the earthquake; 6 . health care professionals due to higher level of exposure and hard-working in apocalyptic conditions, particularly those working in emergency units and resuscitation departments; 7. those punished due to breaking isolation measures, 8. poor people with low social status; 9. those who are highly anxious about their health, known as hypochondriacs, 10. psychiatric patients who could not give appropriate follow up treatment during pandemics. In this article we want to emphasize the role of psychodynamic approach during COVID-19 pandemic. It is crucial to be proactive and anticipate problems before they happen.

\section{MENTAL HEALTH RISKS IN A TIME OF PANDEMIC}

It is quite clear that the COVID-19 pandemic affects not only physical health, but also mental health and individual and collective behavior and way of life changing priorities and challenging health care system functioning (Fiorillo \& Gorwood 2020). This pandemic is not only about virus infecting people all over the world. It is also caused and contained by the way 
that people behaved so it is complex psychodynamic story. Pandemics goes under control only when people agree to behave in particular way such as covering their faces with masks, washing their hands, complying with physical distancing, etc. (Taylor 2020). If people from various reasons refuse to practice preventive measures, than the pandemic will explode. Social and group psychodynamic is also very important, how community and government react as well as how countries cooperate and $\mathrm{UN}$ and $\mathrm{WHO}$ operate and function.

Due to huge mental health risks, the number of people who need psychiatric help is going to continually increase due to fear of uncertainty, death, loss of job, drastic changes of life style, stigmatization, isolation, separation from family and beloved persons, etc. For example, Wang et al. (2020) have observed that during the initial phase of the COVID-19 outbreak in China, more than half of the participants from the general population rated the psychological impact as moderateto-severe, and about one-third reported moderate-tosevere anxiety. Also, Yao et al. (2020) have recently pointed out that mental health patients could be more influenced by the emotional responses brought on by the COVID-19 pandemic, resulting in worsening of an already existing mental health issues because of high susceptibility to stress compared with the general population. To fully understand the mental health repercussions of the COVID-19 pandemic, it is important to consistently examine emotional responses caused by the ongoing crisis, with the emphasis on the impact of fear that people are facing during the ongoing crisis, as we are nowadays continuously witnessing numerous forms of fear-induced behavior. Fear, as one of the primary emotions in humans, can be beneficial in activating stress response and thus helpful in dangerous situations, but can also be a threat when prolonged, serving as a key component in possible development of various psychiatric disorders (Garcia 2017). The medical (health-care) workers who are caring for individuals who are either severely ill, feel scared, or experiencing bereavement are themselves exposed to trauma. Mental health workers are also at risk of getting infected, and they carry a large burden in the clinical treatment and prevention activities in hospitals and community settings. The challenges and stress they experience could trigger different mental disorders, including anxiety and affective disorders. Indeed, some experts believe that maintaining mental health of the medical staff is necessary to better control infectious diseases, although the best approach to this during the epidemic season still remains unclear (Chen et al. 2020). The special emphasis is because of possible development of posttraumatic stress disorder. New knowledge's has lead to more holistic treatment in psychiatry and psychological medicine (Jakovljević 2008, Jakovljević 2017). According to the psychoanalytic view, reaction on virus pandemic is not necessarily a rational process. In many cases, such process appears to be a retrospective "rationalizing" of decisions already made unconsciously by new situation. Thus, intensive psychic reaction can be considered as the output of a process that emphasizes the "dark side" of virus pandemic, integrating the unconscious and fantasies with conscious contents. It is very important to improve public awareness of prevention and intervention strategies by providing daily updates about surveillance and active cases in social media. In this crisis situation, mental health professionals should use internet and social media to share strategies for dealing with psychological stress. Psychodynamic understanding of human pain and psychological problems should be useful in the process of communication between health professionals and general population.

\section{THE USE OF PSYCHODYNAMIC DURING PANDEMIC}

According to some authors COVID-19 is changing our psychology and our behavioral immune system to be morally vigilant and value conformity and obedience over eccentricity or rebellion (Robson 2020). The psychodynamic processes are important for understanding and managing individual and group/public mental health problems and behavior associated with pandemics such as the spreading panic, stigmatization, xenophobia, maladaptive behaviors, defensive reactions, under-reactions, and over-reactions, socially disruptive behavior, hoarding... The World Health Organization (WHO) and the national leading crisis groups consistently encouraged people to socially/physically distance themselves from others and to frequently and thoroughly wash their hands. These concrete steps should have been taken to mitigate the spread of the virus in community and preventing the health-care system from becoming overwhelmed. Individuals and communities who conform to those recommendations served a public health service, and those who violated these recommendations put themselves and others at risk of disease. Unfortunately, many citizens dismissed the seriousness of the COVID-19 pandemic. To understand why many people have not changed their behavior, we need to verify complex contents of unconscious. Psychodynamic approaches to working with problematic behavior focus on a number of different factors that may influence or cause symptoms, such as early childhood experiences (particularly level of attachment to parents), current relationships and the things people do (often without being aware of it) to protect themselves from upsetting thoughts and feelings that are the result of experiencing a stressful event (defense mechanisms). Unlike cognitive-behavioral theory, psychodynamic places a large emphasis on defense mechanisms and unconscious mind, where upsetting feelings, urges, and thoughts that are too painful for us to directly look at are housed. Even though these painful feelings and thoughts are outside of our awareness, they still influence our behavior in many ways. Disavowal is a common defense mechanism that may be used to protect people 
from something that they don't have the ability to cope with. Health professionals (or therapists) may notice that a patient is expressing anger and putting blame on family members (specific family interactions in time of pandemic) even though they haven't done anything to deserve it. In this case, the therapist may interpret this behavior as a sign that the patient is actually upset with and blaming other for the stressful event. Since this anger and guilt is too difficult to cope with (psychological atmosphere during pandemic is turbulent), it is expressed toward others. This defense mechanism is referred as displacement. Denial is defense mechanism which is presented at start of the crisis. People explicitly refusing to acknowledge the threatening reality even when presented with indisputable data (e.g. some other people have problems, not in my town). Denial may give the respondent some time to evaluate the meaning and seriousness of the threatening reality before reacting to it. Suppression means intentionally engaging distractions to eliminate from consciousness any thoughts of the threatening reality. Grandiosity is also presented in crisis when means exaggerated feeling of power or influence over the threatening reality (so-called narcissistic defense). Narcissistic defenses are those processes whereby the idealized aspects of the self are preserved, and its limitations denied. They tend to be rigid and totalistic and could create negative emotional atmosphere. They are often driven by feelings of shame (and guilt) on conscious or unconscious level. Recognition of narcissistic defenses should be useful in working with psychologically difficult patients. Idealization includes ascribing power or influence to an existent or imaginary "savior" (doctor or nurse in crisis time). Projection is based on incorrectly attributing to others any objectionable thoughts or actions (putting blame on others). Splitting refer to fragmenting, isolating, and focusing on only certain elements of the threatening reality, instead of considering the complexity brought about by the crisis as a coherent whole. Stressful event or trauma could cause dissociation or an altered state of consciousness. Great emotion or traumatic event could cause a split in consciousness whereby the ego repressed the traumatic experience. Dissociation obscures the traumatic event that is almost always behind the split in consciousness, and that split, generally, does not become apparent unless a comprehensive history is obtained from a patient (trauma may be detached from conscious memory or denied). Dissociation is difficult to assess as symptoms tend to mask and present as other pathologies. In dissociation, the ego withdraws or fragments to protect the person from the unbearable. A Jungian perspective of COVID-19 pandemic is very complex. In Archetype, Attachment, Analysis: Jungian Psychology and the Emergent Mind, Jean Knox (2003) presented an attachment theory approach to trauma and dissociation. She stated that the main purpose of defenses is affect regulation, with the primary method being through distance regulation (association to social distance during recent COVID-19 crisis). The relationship between caregiver and the child or the actual experience as well as the internal working model builds upon relational and interpersonal intrapsychic activity including the development of defenses. Knox emphasized that relational experiences are stored in implicit memory as generalized patterns. The defensive patterns are also stored as internal working models. They are not habitual but rather are symbolic, representational, and intrapsychic models that, although they remain unconscious. Knox explained the effects of cortisol release in the brain: when high levels of cortisol are repeatedly freed into the blood stream, a malfunction may occur in the hippocampal memory function. This could cause some memory loss following stressful event or trauma. Knox considered it probably impossible to determine if the memory loss is due to dissociation or high exposure levels of cortisol. Knox postulated that the defensive avoidance of memory might lead to hippocampal atrophy. Alexithymia (which means "no words for feelings") should be an indicator of dissociation. According to psychodynamic approach there are three types of unconscious: individual, family and collective. Collective unconscious refers to structures of the unconscious mind which are shared among beings of the same species. It is a term coined by Carl Gustav Jung. Jung considered the collective unconscious to underpin and surround the unconscious mind, distinguishing it from the individual unconscious of Freud. According to Jung, the human collective unconscious is populated by instincts, as well as by archetypes: universal symbols such as The Great Mother, the Wise Old Man, the Shadow etc. Jung proposed that everyone's personality contains elements of four major archetypes and these archetypes provide models for our behavior and influence the way we think and act. Jung labeled these archetypes: the Self, the Persona, the Shadow and the Anima/Animus. COVID-19 pandemic should be assessed as phenomenon of collective unconscious associated to archetypes. Every negative aspect of archetype has symbolic meanings and potential positive challenge. In the year 2020 the world needs to psychologically learn from earlier experiences because this is not the first time that pandemic event is presented. The role of psychodynamic is very important in psychosocial service system, including telephone-based and Internet-based counseling for health-care staff, patients, family members, and the public. In our previous articles (Marčinko 2011, Marčinko et al. 2013, 2015, 2018) we have elaborated that psychodynamic concepts of mentalization and intersubjectivity have direct implications for problem solving and crisis oriented clinical situations, and that the notion of the "third" (meta-position) is particularly useful in understanding what happens in the patient-doctor relationship. We believe that including psychodynamic approach in the national public and mental health emergency system will empower Croatia and the world during (and after) COVID-19 pandemic crisis. 


\section{THE USE OF PSYCHODRAMA TOOLS DURING PANDEMIC}

Since psychodrama as a psychotherapeutic technique has evolved from the psychodynamic concepts, most notably transference and countertransference, defense mechanisms, object relations theory and such, it makes sense to talk about psychodramatic ways of providing help during the global threat of the corona virus.

The central tool of psychodrama is a role, and role reversal constitutes the basic technique and the foundations of psychodrama. One of the goals of psychodrama is to play the role of another person, thing, phenomenon and see and feel oneself and others from a different perspective. Role playing also means seeking opportunities, trying out new situations and making decisions in mid-action - all of which are very useful ways of practicing social skills.

However, it is well known that during a pandemic (due to quarantine, self-isolation, testing for the presence of the virus, hospitalization, etc.) human roles become reduced to the bare structure of a person's social atom. This means that an (infected) person ceases to be, for example, a neighbor, a teacher, somebody's uncle, a good friend and such. Instead, he becomes a (potential) patient. This is why the role theory in psychodrama operates with the so called role expansion. It allows a member of a psychodrama group to train entering different roles which enhances his imagination, creativity and vitality. The founder of psychodrama J.L. Moreno thought that every personality is made up of a series of roles and that the goal is to develop as wide a repertoire as possible. Since the role repertoire is significantly diminished during a pandemic, it is very important to be aware of the new roles, as well as develop them and work through them. These roles may be containment roles, or trauma (of the pandemic) based roles, and when a patient employs them together, new, transformative roles appear. These are new, personalized roles utilized by every member of the group (patient) to create better connection with oneself and others, to create personal autonomy which will help, in a new and different way. to repair the painful experience. This way he changes his behavior, which, in turn, causes the environment to change.

Psychodrama experiential psychotherapy can be successfully applied to the complex web of trauma caused by coronavirus, earthquake and uncertain postpandemic future, in particular if it is utilized expertly and if it relies on the theory for support. The end goal is to establish the sense of personal control over the situation using empowerment techniques while preventing retraumatization.

\section{RESILIENCE, POSTTRAUMATIC GROWTH AND ANTI-FRAGILITY IN TIME OF COVID-19}

The outbreak of coronavirus disease 2019 (COVID19) may be stressful for people. Fear and anxiety about a disease can be overwhelming and cause strong emotions in adults and children. Coping with stress will make you, the people you care about, and your community stronger. Our attention is focused primarily on two topics: 'mental health problems regarding pandemic' and 'post-traumatic growth,' which is defined as positive psychological change that is experienced as a result of trauma or adversity. These are great ambitions given to the sometimes disturbed and weak states that survivors of stress experience. Affected individuals report phenomena that may be called 'post-traumatic growth' (Zoellner \& Maercker 2006). To understand the ingredients of adaptation to traumatic events, it is necessary to examine the process of adaptation (recent and earlier experiences). Change in behavior (or symptoms) requires getting in touch with person and "working through" those painful unconscious feelings. To do this, the therapist will assist the patient in recognizing the defense mechanisms being used, what they are being used for (to avoid painful feelings in the unconscious mind stemming from a traumatic experience), and connecting with and appropriately releasing those feelings and thoughts that were previously being avoided. Defense mechanisms associated to COVID-19 pandemic should be more immature than usually. Anxiety change some persons to lower level of functioning which is assessed by defense mechanisms. It is known that stressful and traumatic experiences, such as the ongoing COVID-19 pandemic, can lead to various acute stress reactions among some people, accompanied by immature defense mechanisms in which the anxiety over a virus outbreak is temporarily alleviated by severe alteration of painful mental contents and/or radical distortion of external reality. More so, the level of psychological symptoms seems to be related to the number of employed neurotic and immature defenses (e.g., displacement, devaluation, projection, acting out, autistic fantasy, somatization) (Santana et al. 2017). The use of defense mechanisms during a global health crisis should be monitored because psychological health is closely related to the ability to appropriately use a variety of defenses in challenging contexts, and a predominant use of immature defenses is a risk factor for the development of different forms of adult psychopathology (Bond \& Perry 2004, Zanarini et al. 2009). Children and young adults should also be assessed from a psychodynamic perceptive given that an increased use of immature defenses and a reduced use of mature defenses have a negative impact on the development of personality (Granieri et al. 2017). Indeed, seminal concepts such as the "rigidity" of personality or the “character armor" (Reich 1933/1979) clearly express the idea that mental health is strictly related to emotional flexibility. Regarding gender differences, it is expected in these globally stressful times that women will use more internalizing defense mechanisms (such as somatization), while men will use more externalizing defenses (such as acting out), a notion in line with early psychoanalytic and clinical observations (Erikson 1964) 
and research findings (Furnham 2012). Vaillant (2011) describes several ways in which immature defenses can be penetrated. Firstly, it can be done through confrontation by supportive peers, or by focused psychotherapy in which, among else, the therapist would interpret the patient's behavior and share this interpretation with the patient. Also, it could be done by improving intrapsychic competence by rendering the individual less anxious and lonely through empathy. These theoretical assumptions are tentatively supported by a small number of research endeavours focusing on how change in defense mechanisms happens over treatment and time, particularly through dynamic psychotherapy, but also appropriate medication (Perry \& Bond 2012, Perry et al. 2020). Psychodynamic approach is associated to different schools. As we mentioned in earlier publication (Jakovljević et al. 2012) seven perspectives model of post-traumatic stress is broadly defined in complementary terms and concepts and overlapping, but distinct constructs. This model provides an transdisciplinary integration of different perspectives and various treatment modalities from psychopharmacotherapy to well-being therapy in order to decrease illness and increase wellness through traumatic experience.

Psychological resilience is the ability to mentally or emotionally cope with a crisis or to return to pre-crisis status quickly. It is a truism that stress experiences can change a person's life (de Terte \& Stephens 2014). Resilience exists when the person uses "mental processes and behaviors in promoting personal assets and protecting self from the potential negative effects of stressors" (Robertson et al. 2015). Public and global mental health promotion may be predicated on the theory of salutogenesis and three key inter-related terms: empathy, coherence and resilience (Jakovljević 2018). In accordance to resilience theory, the outcomes of stress or traumatic event exposure are not all necessarily negative. It is important identifying factors that confer protection to individuals or aid their recovery and those that contribute to positive stressrelated developments in the post-event period. The process of post-traumatic adjustment includes resilience and coping with unconscious and conscious contents. But meaningful connection can occur even from the recommended six feet of social distance between you and your neighbor - and it begins with compassion (COVID-19 pandemic recommendations). Compassion is the intention to be of benefit to others and it starts in the mind. The WHO Mental Health Action Plan 20132020 is based on the vision of a world in which mental health is valued, promoted and protected, and has four objectives: to improve leadership and governance, health and social care, promotion and prevention, and information and research. Practicing public and global actions that promote and educate empathy, coherence and resilience may significantly help achieving equality for mental health in the $21^{\text {st }}$ century and improving public and global mental health (Jakovljević 2018). The aim of earlier paper (Jakovljević et al. 2019) emphasized understanding and creative cooperation between religions and nations in promotion of public and global mental health, research, patient care and education. As a measure of psychological adaptation, defense mechanisms have been studied in the context of resilience. Mature defense mechanisms, such as humor, altruism, anticipation and self-observation can improve resilience capacity during crisis. Humor is defense mechanisms that should enhance resilience during COVID-19 crisis. Emphasizing the amusing or ironic aspects of the conflict or stressor, thereby diffusing tension or conflict. Altruism is also mature defense mechanism and acting by fulfilling the needs of others, in part as a way of fulfilling own needs. Anticipation is defense mechanisms and creates preparation for future difficulties by considering solutions and anticipating emotional reactions to future problems. Self-observation implies a reflection of own thoughts, feelings, motivation and behavior, seeing self as others do in interpersonal situations. Important psychodynamic concept regarding psychic resilience is narcissistic vulnerability. Narcissistic injuries are frequently presented during pandemic crisis which mean that narcissistic vulnerability is one of the core factors in the context of resilience. In our previous work (Marčinko et al. 2014), we have found that narcissistic vulnerability is more strongly related to depressive symptoms than narcissistic grandiosity, and dysfunctional perfectionism represents one of the underlying mechanisms of this relationship. The health professionals (or therapists) and patient can begin to break down these unhealthy mechanisms and bring insight to the underlying problem. Through this insight, the patient can then begin to work through those painful feelings in a healthier and more appropriate manner. Choice of defense mechanisms is involuntary, but socalled "mature defenses" (eg, sublimation and humor) rather than "immature defenses" (eg, projection and hypochondriasis) can make an enormous difference in mental health resilience. Successful, resilient antifragile community without scapegoating and conspiracy as well as successful, resilient and-anti-fragile families, depend on their members' ability to repair relationships when there have been ruptures and conflicts between their members. The foundation to resolving the crucial problems is to use empathy, restorative justice and mentalization to understand the other party what means that both or all sides have values, dignity and respects and their attitudes and actions have meaning (see Jakovljevic et al. 2020).

\section{CONCLUSION}

Understanding how crisis influence an individual's reactions to stressful events (and vice versa) is important in order to create meaningful and effective interventions. Despite the immense effort by health workers to address stress- and trauma-related disorders in the course of COVID-19 pandemic, a large proportion of the people affected by disorder do not have access to 
psychological or psychiatric treatment. Psychic reaction can be considered as the output of a process that emphasizes the "dark side" of virus pandemic, integrating the unconscious and fantasies with conscious contents. We believe that including psychodynamic approach in the national public and mental health emergency system will empower Croatia and the world during (and after) COVID-19 pandemic crisis.

\section{Acknowledgements: None.}

\section{Conflict of interest: None to declare.}

\section{Contribution of individual authors:}

Darko Marčinko: idea, concept and design of the article, writing manuscript, approval of the final version.

Miro Jakovljević: concept and design of the article, literature searches, writing manuscript, approval of the final version.

Nenad Jakšić \& Sarah Bjedov: literature searches, writing manuscript, approval of the final version.

Aleksandra Mindoljević Drakulić: writing manuscript, approval of the final version.

\section{References}

1. Bond M, Perry JC: Long-term changes in defense styles with psychodynamic psychotherapy for depressive, anxiety, and personality disorders. Am J Psychiatry 2004; 161:1665-1671

2. Chen Q, Liang $M, L i Y$, et al: Mental health care for medical staff in China during the COVID-19 outbreak. Lancet Psychiatry 2020; 7:e15-e16

3. de Terte, I, Stephens C: Psychological Resilience of Workers in High-Risk Occupations. Stress and Health 2014; 30:353-355

4. Erikson EH: Inner and outer space: reflections on womanhood. In Lifton RJ (ed): The Woman in America, 126. Beacon Press, 1964

5. Fiorillo A \& Gorwood P: The consequencies of the COVID19 pandemic on mental health and implications for clinical practice. Editorial. European Psychiatry, April 2020, pp. 1-4. doi: https://doi.org/10.1192/eurpsy.2020.35

6. Furnham A: Lay understandings of defence mechanisms: the role of personality traits and gender. Psychol Health Med 2012; 17:723-734

7. Garcia R: Neurobiology of fear and specific phobias. Learn Mem 2017; 24:462-471

8. Global Ministerial Mental Health Summit: Global Declaration on Achieving Equality for Mental Health in the 21st Century, 2018

9. Granieri A, La Marca L, Mannino G, Giunta S, Guglielmucci F, Schimmenti A: The Relationship between Defense Patterns and DSM-5 Maladaptive Personality Domains. Front Psychol 2017; 8:1926

10. Jakovljević $M$, Bjedov $S$, Jakšić $N$ \& Jakovljević I: COVID-19 pandemic from the perspective of public and global mental health. Psychiatr Danub 2020; 32:6-14
11. Jakovljević M, Brajković L, Jakšić N, Lončar M, AukstMargetić B \& Lasić D: Posttraumatic stress disorders (PTSD) from different perspectives: a transdisciplinary integrative approach. Psychiatr Danub 2012; 24:246-255

12. Jakovljević M, Kurjak A, Jerković A, Hasanović A \& Nikić $M$. Spirituality, religiosity and nationalism from the perspective of public and global mental health. Psychiatr Danub 2019; 31:382-391

13. Jakovljevic M: Empathy, sense of coherence and resilience: Bridging personal, public and global mental health and conceptual synthesis. Psychiatr Danub 2018; 4:380384

14. Jakovljević M: Public and global mental health promotion for empathic civilisation: a new goal of Psychiatria Danubina. Psychiatr Danub 2016; 28:312-314

15. Jakovljević M: Resilience, Psychiatry and Religion from Public and Global Mental Health Perspective - Dialogue and Cooperation in the Search for Humanistic Self, Compassionate Society and Empathic Civilization. Psychiatr Danub 2017; 29:238-244

16. Jakovljević M: Transdisciplinary holistic integrative psychiatry - a wishfull thinking or reality? Psychiatr Danub 2008; 20:341-8

17. Knox J: Archetype, attachment, analysis: Jungian psychology and the emergent mind. New York, NY: Routledge, 2003

18. Marčinko D, Jakšić N, Ivezić E, Skočić M, Surányi Z, Lončar M, Franić T, Jakovljević M: Pathological Narcissism and Depressive Symptoms in Psychiatric Outpatients: Mediating Role of Dysfunctional Attitudes. J Clin Psychol 2014; 70:341-52

19. Marčinko D, Jakšić N, Skočić M, Franić T: Mentalization and psychopharmacotherapy in patients with personality and eating disorders. Psychiatr Danub 2013; 25:320-3

20. Marčinko D, Radanović Ćrić S, Šeparović Lisak M, Rudan D, Jeleč $V$, Bilić $V$ : The role of psychodynamic and personality assessment in psychopharmacotherapy of suicidal psychotic patients. Psychiatr Danub 2018; 30(Suppl 4):228-230

21. Marčinko D: Intersubjectivity and psychopharmacotherapy in the treatment of chronically suicidal patients. Psychiatr Danub 2011; 23:325-7

22. Marčinko D: Psychodynamic and psychopharmacotherapy in the treatment of difficult patients with personality and eating disorders. Psychiatr Danub 2015; 27:330-3

23. Perry JC, Banon E, Bond M: Change in Defense Mechanisms and Depression in a Pilot Study of Antidepressive Medications Plus 20 Sessions of Psychotherapy for Recurrent Major Depression. J Nerv Ment Dis 2020; 208:261-268

24. Perry JC, Bond M: Change in defense mechanisms during long-term dynamic psychotherapy and five-year outcome. Am J Psychiatry 2012; 169:916-25

25. Reich W: Character Analysis. Farrar, Straus and Giroux, New York, 1933/1979

26. Robertson IT, Cooper CL, Cary L, Sarkar M, Curran T. Resilience training in the workplace from 2003 to 2014: A systematic review. Journal of Occupational and Organizational Psychology 2015; 88:533-562

27. Robson D: The fear of corona virus is changing our psychology. BBC Future, April 2, 2020 bbc.com/future/article

28. Santana MRM, Zatti C, Spade ML, Malgarim BG, Salle E, Piltcher R, Ceresér KMM, Bastos AG \& Freitas LH: 
Acute stress disorder and defense mechanisms: a study of physical trauma patients admitted to an emergency hospital. Trends in Psychiatry and Psychotherapy 2017; 39:247-256

29. Shultz JM, Baingana F, Neria Y: The 2014 Ebola outbreak and mental health: current status and recommended response. JAMA 2015; 313:567-68

30. Taylor S: I've spent years styding the psychology of pandemics. This is what you need to know about Covid-19. British Columbia, 17 March, 2020 https://www.psychwre.com

31. Vaillant GE: Involuntary coping mechanisms: a psychodynamic perspective. Dialogues Clin Neurosci 2011; 13:366-70
32. Wang C, Pan R, Wan X, Tan Y, Xu L, Ho CS, Ho RC: Immediate Psychological Responses and Associated Factors during the Initial Stage of the 2019 Coronavirus Disease (COVID-19) Epidemic among the General Population in China. International Journal of Environmental Research and Public Health 2020; 17:1729

33. Yao H, Chen JH, Xu YF: Patients with mental health disorders in the COVID-19 epidemic. Lancet Psychiatry 2020; 7:e21

34. Zanarini MC, Weingeroff JL, Frankenburg FR: Defense mechanisms associated with borderline personality disorder. J Pers Disord 2009; 23:113-121

35. Zoellner T, Maercker A: Posttraumatic growth in clinical psychology - a critical review and introduction of a two component model. Clin Psychol Rev 2006; 26:626-53

\section{Correspondence:}

Ass. Professor Darko Marčinko, MD, PhD, psychiatrist-psychotherapist

Department of Psychiatry and Psychological Medicine,

University Hospital Center Zagreb

Kišpatićeva 12, 10000 Zagreb, Croatia

E-mail: niarveda@gmail.com 